\title{
Intervista al Poeta Mario Luzi
}

\author{
Vera Lúcia de OLIVEIRA*
}

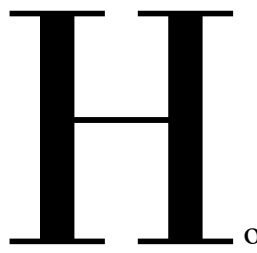

incontrato Mario Luzi il 10 ottobre del 1991, in un freddo pomeriggio di autunno a Perugia, in una sera in cui il vento gelido e forte spazzava dalle strade i passanti desiderosi di arrivare presto a casa. Come altre interviste da me fatte a poeti italiani contemporanei, anche questa è stata realizzata nell'ambito degli incontri "Poesia a Palazzo dei Priori", che il Gruppo di Sperimentazione e Ricerca Poetica Il Merendacolo ha organizzato a Perugia dal 1989 al 2009.

* Università degli Studi di Perugia 
In un'intervista lei ha affermato che la poesia oggi torna ad essere necessaria al mondo, ossia torna ad avere un ruolo importante nella società. Allo stesso tempo, però, constatiamo che le persone leggono sempre meno poesia e che la società civile, politica, non riconosce nessun ruolo al poeta. Come superare questo scarto, come il poeta potrà rivelare, e a chi, questo terribile momento di alienazione che tutti viviamo, momento di violenza, di allontanamento dell'essere da sé stesso? Che cosa potrà fare il poeta?

La poesia che è stata per molto tempo un po' celebrativa e cioè laudativa dell'esistente, oggi, dopo circa un secolo di conflittualità, si è assunta l'incarico, il ruolo di organizzare il caos nel regime costante della nostra storia. In altre parole, di riconoscere il caos e anche, perché lo riconosce, di ordinarlo e di esprimerlo. Questo mi pare che sia un potere che la poesia ha e che esercita per natura o per vocazione. Alcuni poeti sono più o meno coscienti di questa facoltà concessa alla poesia e agiscono più o meno coerentemente con questa consapevolezza.

Quanto poi alla rispondenza del pubblico che lei lamenta insufficiente, è vero che si legge forse sempre meno, ma si legge meno poesia come si legge meno qualunque altra cosa. Cioè si è sostituita una lingua convenzionale, comoda, più che altro visiva, alla vera interpretazione e valutazione del linguaggio. Però se è vero questo, è vero anche che chi legge poesie o scritture attuali sente di essere chiamato a qualcosa, sente di non essere intrattenuto gratuitamente, almeno nei veri scrittori. Essere chiamati a qualcosa, cioè a una lettura, a un'interpretazione reale di tutto quello che viene proposto. Ed ecco la contraddizione. Il poeta oggi ha una scarsa importanza, è in fondo una figura che non ha il prestigio sociale che poteva aver avuto nel passato, quando in fondo non faceva nulla di utile. E oggi, che credo che il poeta sia utilissimo, necessario a riconnettere con l'umano quello che si è disperso, il poeta non ha un ruolo, non ha un posto nella società come tale, come poeta, come lo ha il banchiere, il professore. Ma questo è uno dei paradossi della nostra epoca, per cui più una cosa è necessaria, meno è apprezzata.

La poesia sarebbe pertanto il linguaggio dell'essenzialità, della discesa nella profondità di esseri e cose. Ma come può la lingua, creazione umana, cogliere questa complessità dell'universo, la metamorfosi continua di tutto? Non ci sono dei momenti in cui sente la limitazione frustrante delle parole?

Sì, la poesia è il linguaggio dell'essenzialità ed è vero che la metamorfosi, il mutamento, possono portare le cose lontano dalla loro genuina essenzialità. Però nel loro mutamento, nella loro trasformazione, le cose autentiche, le cose vere, passano per quella fase essenziale da cui 
sono emerse originariamente e allora anche questa difficoltà, che lei giustamente afferma, che ha la parola, che hanno gli scrittori che credono, che sentono la pulsazione di un linguaggio inadeguato, anche questa difficoltà finisce per essere in un certo senso superata. C'è un momento in cui si ristabilisce una coincidenza fra la cosa e la parola, fra la verità, la sostanza, e il nome. E allora la convenzione, la civiltà, questa scorciatoia dei segni convenzionali, viene superata. C'è questo momento ed è un momento poetico.

\section{È il momento del miracolo?}

Sì, è proprio il momento del miracolo.

In una sua conferenza a Napoli, lei ha affermato che "la nascita di una parola esige l'uccisione sacrificale di altre parole divenute inservibili per usura ed estinzione di significato". Per lei, allora, il poeta oggi è una specie di sacerdote che ha il dono e la missione di rimettere in contatto cose e parole vive?

Non oggi, sempre. Ma non occorre solennizzare a tal punto il suo ruolo. La sua funzione è oggettivamente quella: e lo è anche a livello artigiano.

In questo fine millennio, il poeta può ancora avere un ruolo nella società? Ma che cos'è il poeta? Una voce discordante, un profeta del futuro?

Sì, ce l'ha, è poco evidente ma molto concreto. Si combatte una grande partita tra la persistenza di valori che credevamo l'umano, l'irrinunciabile, e le tentazioni di un'ab-umanità tecnologica invadente. Drammatizzare questo conflitto e umanizzare questo trapasso spetterà, penso, soprattutto alla poesia.

\section{Quali progetti ha in questo momento?}

Vorrei fare ancora qualcosa per il teatro. Ma a un certo punto è la vita, nella sua umiltà quotidiana, il solo regista. 